\title{
Cutoff and Target Values for Intra-Abdominal Fat Area for Prevention of Metabolic Disorders in Pre- and Post-Menopausal Obese Women Before and After Weight Reduction
}

\author{
Ryosuke Shigematsu, PhD; Tomohiro Okura, $\mathrm{PhD}^{*}$; Syuzo Kumagai, $\mathrm{PhD}$ **; \\ Yuko Kai, $\mathrm{PhD}^{* *},+\neq ;$ Teruo Hiyama, $\mathrm{MD}^{\dagger}$; Haruka Sasaki, $\mathrm{MD}^{\dagger \dagger}$; \\ Hitoshi Amagai, MSc $\leftleftarrows$; Kiyoji Tanaka, $\mathrm{PhD}^{*}, \S$
}

\begin{abstract}
Background The Japan Society for the Study of Obesity originally proposed a cutoff value of $>100 \mathrm{~cm}^{2}$ for the intra-abdominal fat area (IFA) as a definition for "visceral fat obesity" in Japanese adults. There are no studies on the cutoff or target values after weight reduction in pre- and post-menopausal women.

Methods and Results In the present study 149 pre-menopausal obese women (PreM, 43.3 years, $\left.27.3 \mathrm{~kg} / \mathrm{m}^{2}\right)$ and 58 post-menopausal women (PostM, 53.9 years, $27.7 \mathrm{~kg} / \mathrm{m}^{2}$ ) participated in a 14-week weight reduction program. The IFA was measured by computed tomography. The program induced significant reductions in body weight $(8.6 \mathrm{~kg}$ in PreM and $7.8 \mathrm{~kg}$ in PostM). The IFA decreased significantly from $80.4 \pm 41.3$ to $50.7 \pm 23.8$ (PreM) and from $115.4 \pm 38.0$ to $75.7 \pm 30.5$ (PostM).

Conclusions The receiver-operating characteristic curve analyses revealed that the appropriate cutoff values were $80 \mathrm{~cm}^{2}\left(\right.$ PreM) and $110 \mathrm{~cm}^{2}$ (PostM) before the program, and after the program the appropriate target values were determined as 60 and $70 \mathrm{~cm}^{2}$, respectively. (Circ J 2006; 70: 110-114)
\end{abstract}

Key Words: Diet; Exercise; Fat body; Menopause; Metabolic syndrome

$\mathbf{T}$ he "visceral fat obesity" refers to the condition of excess intra-abdominal fat (IF), which places people having this type of excess fat at high risk for obesity-related metabolic disorders, such as hyperglycemia and dyslipidemia. The Japan Society for the Study of Obesity (JASSO) ${ }^{1}$ originally defined visceral fat obesity in Japanese as having an IF area (IFA) $>100 \mathrm{~cm}^{2}$ and indicated that such people tend to have 1 or more metabolic disorders! Nakamura et al reported that approximately $62 \%$ of patients with coronary artery disease have an IFA $=100 \mathrm{~cm}^{2}$ or more, and Banno et al found that sleep-disordered breathing was closely associated with obesity?

JASSO used a cross-sectional study design to validate the cutoff value for IFA of $100 \mathrm{~cm}^{2}$ for the diagnosis of visceral fat obesity, but intervention studies for assessing an appropriate target value that can be used for people who reduce their IF significantly have been lacking, and it is unclear whether, or at what point, decreasing IF improves metabolic disorders.

(Received August 26, 2005; revised manuscript received October 19, 2005; accepted November 4, 2005)

Faculty of Education, Mie University, Tsu, *Graduate School of Comprehensive Human Sciences, University of Tsukuba, Tsukuba, **Institute of Health Science, Kyushu University, Fukuoka, ${ }^{\dagger}$ Division of Cardiology, Higashi Toride Hospital, Toride, "Department of Internal Medicine, Chikushi Hospital, Fukuoka University, Chikushino, \#epartment of Orthopedic Surgery, Moriya Keiyu Hospital, Moriya, \#Physical Fitness Research Institute Meiji Yasuda Life Foundation of Health and Welfare, Tokyo and \$Tsukuba Advanced Research Alliance, University of Tsukuba, Tsukuba, Japan

Mailing address: Ryosuke Shigematsu, $\mathrm{PhD}$, Faculty of Education, Mie University, 1577 Kurimamachiya, Tsu, Mie 514-8507, Japan.

E-mail: rshige@edu.mie-u.ac.jp
There are several studies of the effects of menopause on the relationship of IF with metabolic diseases. Excess IF deposition is more prevalent in post-menopausal women than in pre-menopausal women ${ }^{4}$ although it occurs more frequently in males of all ages? Hunter et $\mathrm{al}^{6}$ and Gower et $\mathrm{al}^{7}$ showed that the IFA and the risk of coronary heart disease (CHD) were positively correlated and that each average in post-menopausal women was higher than that in pre-menopausal women. The results of the study by Rebuffe-Scrive et $\mathrm{al}^{8}$ suggest that one of the reasons for this phenomenon is the more pronounced activation of lipoprotein lipase in the omental adipose tissue of postmenopausal women than in that of pre-menopausal women. The cutoff value for the IFA derived by $\mathrm{JASSO}^{1}$ was defined using a combination of pre- and post-menopausal women; the standards were, therefore, not established while considering the presence of menopause.

Based on these results, the current study assesses JASSO's visceral fat obesity IFA cutoff value of $100 \mathrm{~cm}^{2}$ in pre- and post-menopausal women and also assesses the IFA target value after a weight reduction program. We tested 2 related hypotheses: (1) the cutoff value would be valid when applied to a group consisting of only pre- or post-menopausal women and (2) it would remain valid in each group after reducing the IFA.

\section{Methods}

Participants

Advertisements were placed in local newspapers and on bulletin boards in Toride City in Ibaraki Prefecture and Abiko City in Chiba Prefecture in Japan to locate potential 
participants with a desire to lose weight. Those who responded to the advertisements were interviewed by telephone. The participants supplied information on demographics, menstrual status, and medical history. They were excluded from the study if their weight had been unstable for the past 6 months, if they had attended any weight reduction programs in the past year, or if they were breast feeding or pregnant. A study physician confirmed if participants were possibly pregnant. Further, the study staff and radiologic technologist explained to all participants that computed tomography (CT) can have deleterious effects. After applying the exclusion criteria to potential participants, the selected participants $(n=220)$ received the details of the study's purpose and protocol. Oral informed consent, following the Helsinki Declaration principles and approved by the Higashi Toride Hospital Review Board, was obtained from each person. We defined "menopause" as the status of no menses for 1 year prior to the study. "Pre-menopause" was used define individuals who were not experiencing menopause. Therefore, the pre-menopausal group consisted of women who declared having menses in the year prior to the study (PreM). The post-menopausal group included those women who had not had menses for more than 1 year prior to the beginning of the study (PostM).

\section{IFA}

We measured the IFA and subcutaneous fat area (SFA) at the level of the umbilicus using cross-sectional CT (SCT-6800TX; Shimadzu, Japan). Scans were performed with the participants in the supine position. Details of the scanning have been reported by Tokunaga et $\mathrm{al}^{9}$ and Yoshizumi et al ${ }^{10}$ Measurements taken before and after the program were conducted at the same time of day by the same technician to minimize technical error. The IFA and SFA were calculated using a computer-software program (FatScan; N2system, Japan) $!^{10}$ The intra-class correlation for repeated IFA determinations in the laboratory (Institute of Health and Sport Sciences, University of Tsukuba) is $0.99(n=30)$.

\section{Obesity-Related Metabolic Disorders}

The obesity-related metabolic disorders were defined as follows: accumulation of IF (waist circumference $\geq 90 \mathrm{~cm}$ in female) plus 2 or more co-morbidities consisting of (i) triacylglycerol (TG) $\geq 150 \mathrm{mg} / \mathrm{dl}$ and/or high-density lipoprotein cholesterol (HDLC) $<40 \mathrm{mg} / \mathrm{dl}$, (ii) systolic blood pressure (SBP) $\geq 130 \mathrm{mmHg}$ or diastolic blood pressure (DBP) $\geq 85 \mathrm{mmHg}$, or (iii) fasting plasma glucose $\geq 110 \mathrm{mg} / \mathrm{dl}{ }^{11,12}$ These biochemical assays were performed on approximately $10 \mathrm{ml}$ of blood drawn from each participant after an overnight fast. The blood assays were analyzed by technicians at the Koto Biken Research Institute in Tsukuba, Japan. Total body composition was assessed by bioelectrical impedance methods ${ }^{13}$ We used the Tanaka formula ${ }^{13}$ to estimate the total body density $(\mathrm{Db})$ and the Brozek formula ${ }^{14}$ to determine the percentage of body fat. The Tanaka formula accurately predicts the total $\mathrm{Db}$ in obese Japanese women $\left(\mathrm{R}=0.903, \mathrm{SEE}=0.0061 \mathrm{~g} / \mathrm{cm}^{3}\right.$, with the hydrodensitometrically determined $\mathrm{Db}$ ). SBP and DBP were taken from the right arm using a mercury manometer after at least a 20-min rest while seated. Cuff sizes were selected based on upper arm girth and length.

Weight Reduction Program

A 14-week weight reduction program was monitored by a physician, dietician, exercise instructors, and graduate school students majoring in exercise intervention. After the baseline assessment, participants received instruction on the diet program, which comprised weekly 90-min diet consultations, at which a diet-recording notebook and several handouts were given to participants to help them adhere to the principles of the daily diet. They were asked to take a well-balanced supplemental food product (MicroDiet; Sunny Health Co, Ltd, Japan) daily as 1 of their meals, preferably as lunch or dinner. The MicroDiet, which includes various amino acids, vitamins, and minerals, was developed for very low-energy diets. To prevent boredom, the MicroDiet was served in 7 flavors: coffee, milk tea, cocoa, yogurt, banana, strawberry, and apple. Participants received packages consisting of 7 meals (each flavor) once a week. The nutritional values for each flavor were slightly different (ie, there was a range for protein (20.6-21.5 g), carbohydrate (15.0-18.1 g), fat (1.6-3.0 g), and energy (169-173 kcal) for each meal). The diet records were obtained from 86 participants (60 in the PreM group, 26 in the PostM group), who were randomly selected. One week before the study, the participants were asked to record everything they had eaten for the 3 days prior to the study. Furthermore, they were asked to record their diets for 3 days during week 7 , the midpoint of the intervention.

The exercise program included 3 weekly 45-min sessions. During the first and second weeks of the 14-week program, exercise sessions consisted mainly of walking and stretching, with the gradual addition of a bench-stepping exercise $^{15}$ as the main element. Thereafter, the exercise session consisted of a 10-min warm-up, 25-min bench stepping, and a 10-min cool-down. The bench stepping targeted an exercise intensity in which the participant's heart rate reached a level 10-15\% higher than the level corresponding to her lactate threshold (LT). The LT was defined as the point at which blood lactate concentration maintained a non-linear increase above the level at rest 16 To determine LT, a series of venous blood samples ( $1 \mathrm{ml}$ each) was drawn from the antecubital vein every minute during a maximal cycling exercise test, which was done with an accompanying electrocardiogram as a baseline assessment. All blood samples were analyzed by the electrochemical enzymatic method using a lactate analyzer (model 23L, YSI Inc, OH, USA). For establishing LT, the log (oxygen uptake) - log (lactate) transformation method was used! 16

Exercise was consistently performed for $45 \mathrm{~min}$ throughout the 14 weeks, but the intensity was progressively increased. In the first 2 weeks, the bench-stepping instructor targeted the intensity as described. After the $3^{\text {rd }}$ week, the instructor progressively increased the intensity by increasing the cadence of the step and adding more dynamic movements. Ratings of the perceived exertion (RPE) ${ }^{17}$ by all participants were also monitored during the bench stepping. Based on their RPE, the instructor moderated the intensity as "somewhat hard" to "hard," which corresponded to $\mathrm{LT}$ or a little above LT! 18

\section{Statistical Analysis}

Differences in variables between the beginning and end of the program were tested in each group by using Student's paired t-tests. Data were analyzed with the SPSS 11.01J statistical software package (SPSS, Chicago, IL, USA), and $\mathrm{P}$-values less than 0.05 were considered statistically significant.

To assess the cutoff value (before weight reduction) and 
Table 1 Baseline Characteristics of Participants

\begin{tabular}{lccc}
\hline \hline & PreM+PostM $(n=207)$ & PreM $(n=149)$ & PostM $(n=58)$ \\
\hline Age $($ years $)$ & $46.2 \pm 8.1$ & $43.3 \pm 6.7(24-57)$ & $53.9 \pm 6.0(45-62)$ \\
Height $(\mathrm{cm})$ & $157.0 \pm 5.2$ & $157.9 \pm 5.1(146.1-171.8)$ & $154.6 \pm 4.9(145.6-165.4)$ \\
Weight $(\mathrm{kg})$ & $67.6 \pm 8.2$ & $68.1 \pm 7.6(53.6-87.6)$ & $66.3 \pm 9.7(50.0-111.3)$ \\
Body mass index $\left(\mathrm{kg} / \mathrm{m}^{2}\right)$ & $27.4 \pm 3.0$ & $27.3 \pm 2.9(21.8-37.3)$ & $27.7 \pm 3.3(20.9-40.7)$ \\
Percent body fat $(\%)$ & $34.6 \pm 4.9$ & $34.1 \pm 4.2(24.9-46.7)$ & $35.9 \pm 6.2(24.1-51.9)$ \\
Intra-abdominal fat area $\left(\mathrm{cm}^{2}\right)$ & $90.2 \pm 43.3$ & $80.4 \pm 41.3(12.2-222.9)$ & $115.4 \pm 38.0(32.3-191.2)$ \\
Subcutaneous fat area $\left(\mathrm{cm}^{2}\right)$ & $252.2 \pm 82.1$ & $250.9 \pm 75.4(103.5-548.0)$ & $255.5 \pm 97.9(90.5-684.0)$ \\
Abdominal circumstance $(\mathrm{cm})$ & $95.7 \pm 8.6$ & $95.1 \pm 8.4(73.8-118.0)$ & $97 \pm 8.9(80.5-131.5)$ \\
\hline
\end{tabular}

Values are means \pm standard deviations (minimum-maximum).

PreM, pre-menopausal obese group; PostM, post-menopausal obese group.

Table 2 Effects of a 14-Week Weight Reduction Program on Anthropometric Variables, Abdominal Fat Area, Metabolic Variables, and Blood Pressures

\begin{tabular}{|c|c|c|c|c|c|c|}
\hline & \multicolumn{2}{|c|}{ PreM +PostM $(n=207)$} & \multicolumn{2}{|c|}{$\operatorname{PreM}(n=149)$} & \multicolumn{2}{|c|}{$\operatorname{PostM}(n=58)$} \\
\hline & Before & After & Before & After & Before & After \\
\hline Weight $(\mathrm{kg})$ & $67.6 \pm 8.2$ & $59.3 \pm 7.4 *(-12 \%)$ & $68.1 \pm 7.6$ & $59.6 \pm 6.9 *(-12 \%)$ & $66.3 \pm 9.7$ & $58.5 \pm 8.5 *(-12 \%)$ \\
\hline Body mass index $\left(\mathrm{kg} / \mathrm{m}^{2}\right)$ & $27.4 \pm 3.0$ & $24.0 \pm 2.7 *(-12 \%)$ & $27.3 \pm 2.9$ & $23.9 \pm 2.6 *(-12 \%)$ & $27.7 \pm 3.3$ & $24.4 \pm 2.9 *(-12 \%)$ \\
\hline Percent body fat (\%) & $34.6 \pm 4.9$ & $29.4 \pm 4.6 * \quad(-15 \%)$ & $34.1 \pm 4.2$ & $28.8 \pm 4.0 * \quad(-15 \%)$ & $35.9 \pm 6.2$ & $31.1 \pm 5.5 *(-13 \%)$ \\
\hline Intra-abdominal fat area $\left(\mathrm{cm}^{2}\right)$ & $90.2 \pm 43.3$ & $57.7 \pm 28.1 *(-32 \%)$ & $80.4 \pm 41.3$ & $50.7 \pm 23.8 *(-31 \%)$ & $115.4 \pm 38.0$ & $75.7 \pm 30.5 *(-34 \%)$ \\
\hline Subcutaneous fat area $\left(\mathrm{cm}^{2}\right)$ & $252.2 \pm 82.1$ & $181.6 \pm 76.9 *(-29 \%)$ & $250.9 \pm 75.4$ & $176.2 \pm 73.2 *(-31 \%)$ & $255.5 \pm 97.9$ & $195.5 \pm 84.6 *(-24 \%)$ \\
\hline Abdominal circumference $(\mathrm{cm})$ & $95.7 \pm 8.6$ & $85.2 \pm 8.6 *(-6 \%)$ & $95.1 \pm 8.4$ & $84.9 \pm 8.0 *(-5 \%)$ & $97.3 \pm 8.7$ & $86.2 \pm 10.0 *(-7 \%)$ \\
\hline Fasting plasma glucose $(\mathrm{mmol} / \mathrm{L})$ & $5.41 \pm 1.13$ & $4.94 \pm 0.68 *(-7 \%)$ & $5.25 \pm 0.86$ & $4.88 \pm 0.68 *(-6 \%)$ & $5.84 \pm 1.57$ & $5.10 \pm 0.67 *(-10 \%)$ \\
\hline Total cholesterol $(\mathrm{mmol} / \mathrm{L})$ & $5.71 \pm 0.95$ & $5.17 \pm 0.89 *(-9 \%)$ & $5.59 \pm 0.95$ & $5.02 \pm 0.83 *(-9 \%)$ & $6.00 \pm 0.89$ & $5.57 \pm 0.92 *(-7 \%)$ \\
\hline Triacylglycerol $(\mathrm{mmol} / \mathrm{L})$ & $1.18 \pm 0.59$ & $0.80 \pm 0.41 *(-23 \%)$ & $1.13 \pm 0.59$ & $0.76 \pm 0.38 *(-24 \%)$ & $1.30 \pm 0.58$ & $0.91 \pm 0.48 *(-21 \%)$ \\
\hline$H D L C(\mathrm{mmol} / \mathrm{L})$ & $1.70 \pm 0.38$ & $1.65 \pm 0.33 *(-1 \%)$ & $1.72 \pm 0.36$ & $1.65 \pm 0.32 *(-2 \%)$ & $1.66 \pm 0.44$ & $1.65 \pm 0.35(+2 \%)$ \\
\hline$S B P(m m H g)$ & $132.4 \pm 18.8$ & $120.6 \pm 16.5 *(-8 \%)$ & $129.9 \pm 17.9$ & $118.6 \pm 15.5 *(-8 \%)$ & $138.7 \pm 19.7$ & $125.8 \pm 17.7 *(-9 \%)$ \\
\hline $\mathrm{DBP}(\mathrm{mmHg})$ & $82.1 \pm 11.7$ & $74.4 \pm 11.0 *(-9 \%)$ & $81.0 \pm 11.5$ & $74.2 \pm 10.5 *(-8 \%)$ & $84.9 \pm 11.9$ & $74.8 \pm 12.4 *(-12 \%)$ \\
\hline
\end{tabular}

Values are means \pm standard deviations (relative change, \%).

PreM, pre-menopausal obese group; PostM, post-menopausal obese group; HDLC, high-density lipoprotein cholesterol; SBP, systolic blood pressure; DBP, diastolic blood pressure.

*Significant intra-group difference $(P<0.05)$.

Table 3 Number and Percentage of Participants That Exceeded Each Criterion of the Metabolic Disorders Before and After Weight Reduction Program

\begin{tabular}{lccccc}
\hline \hline & \multicolumn{2}{c}{ PreM $(n=149)$} & & \multicolumn{2}{c}{ PostM $(n=58)$} \\
\cline { 2 - 3 } \cline { 5 - 6 } & Before & After & & Before & After \\
\hline High abdominal circumference & $112(75 \%)$ & $37(25 \%)$ & & $50(86 \%)$ & $18(31 \%)$ \\
High triacylglycerol and/or low HDLC & $27(18 \%)$ & $6(4 \%)$ & & $11(19 \%)$ & $6(10 \%)$ \\
High triacylglycerol & $26(17 \%)$ & $5(3 \%)$ & & $9(16 \%)$ & $5(9 \%)$ \\
Low HDLC & $3(2 \%)$ & $3(2 \%)$ & & $4(7 \%)$ & $2(3 \%)$ \\
High systolic and/or diastolic blood pressure & $80(54 \%)$ & $36(24 \%)$ & & $41(71 \%)$ & $23(40 \%)$ \\
High systolic blood pressure & $74(50 \%)$ & $33(22 \%)$ & & $39(67 \%)$ & $23(40 \%)$ \\
High diastolic blood pressure & $52(35 \%)$ & $22(15 \%)$ & & $28(48 \%)$ & $8(14 \%)$ \\
High fasting plasma glucose & $11(7 \%)$ & $6(4 \%)$ & & $14(24 \%)$ & $6(10 \%)$ \\
\hline
\end{tabular}

Abbreviations see in Table 2.

the target value (after weight reduction) for IFA, receiveroperating characteristic (ROC) curve analysis was applied to the data derived from the IFA and the number of metabolic disorders. By provisionally varying the cutoff/target values of IFA, we calculated the sensitivities and specificities for each value. Sensitivity was defined as the proportion of participants having a given disorder who also had an IFA equal to or greater than the provisional value to all participants having a given disorder. Specificity was defined as the proportion of participants having no disorders who had an IFA that fell below the provisional value to all participants having no disorders. The sensitivities and specificities were calculated for every $10 \mathrm{~cm}^{2}$ of IFA from 30 to $140 \mathrm{~cm}^{2}$. At each $10 \mathrm{~cm}^{2}$ provisional value, the sensitivity was multiplied by the specificity, and the point having the maximum product of sensitivity $\times$ specificity was considered to be the most valid cutoff/target value.

\section{Results}

Of the 220 women originally enrolled in this study, 13 dropped out because they moved out of the area, needed to care for a family member, or felt fatigued. Consequently, 207 women completed the study (Table 1), and attendance averaged $92 \%$ (range $83-100 \%$ ).

There were significant decreases in the anthropometric variables, IFA, SFA, metabolic variables, and blood pressures in each group (Table 2). Total body composition analysis revealed that the reduction in body weight was mostly from loss of body fat. The reduction in fat-free mass 
Table 4 Sensitivities and Specificities From Each Provisional Cutoff/Target Value of Intra-Abdominal Fat Area (IFA)

\begin{tabular}{|c|c|c|c|c|c|c|c|c|}
\hline \multirow{3}{*}{$\begin{array}{l}\text { Cutoff/target value } \\
\left(I F A, \mathrm{~cm}^{2}\right)\end{array}$} & \multicolumn{4}{|c|}{ PreM } & \multicolumn{4}{|c|}{ PostM } \\
\hline & \multicolumn{2}{|c|}{ Before } & \multicolumn{2}{|c|}{ After } & \multicolumn{2}{|c|}{ Before } & \multicolumn{2}{|c|}{ After } \\
\hline & Sensitivity & Specificity & Sensitivity & Specificity & Sensitivity & Specificity & Sensitivity & Specificity \\
\hline 30 & 0.96 & 0.04 & 0.93 & 0.22 & 1.00 & 0.00 & 1.00 & 0.06 \\
\hline 40 & 0.91 & 0.23 & 0.87 & 0.42 & 1.00 & 0.06 & 1.00 & 0.11 \\
\hline 50 & 0.89 & 0.37 & 0.73 & 0.57 & 0.98 & 0.12 & 1.00 & 0.3 \\
\hline 60 & 0.80 & 0.47 & $\underline{0.67}$ & 0.71 & 0.95 & 0.18 & 0.91 & 0.40 \\
\hline 70 & 0.72 & 0.55 & 0.33 & 0.82 & 0.93 & 0.29 & 0.91 & 0.63 \\
\hline 80 & 0.63 & 0.74 & 0.27 & 0.89 & 0.90 & 0.41 & 0.82 & 0.66 \\
\hline 90 & 0.53 & 0.81 & 0.07 & 0.96 & 0.88 & 0.59 & 0.64 & 0.72 \\
\hline 100 & 0.45 & 0.89 & 0.07 & 0.98 & 0.85 & 0.59 & 0.64 & 0.79 \\
\hline 110 & 0.40 & 0.95 & 0.07 & 0.99 & 0.73 & 0.71 & 0.45 & 0.96 \\
\hline 120 & 0.27 & 0.96 & 0.07 & 0.99 & 0.56 & 0.82 & 0.36 & 0.98 \\
\hline 130 & 0.21 & 0.97 & 0.07 & 1.00 & 0.41 & 0.94 & 0.18 & 1.00 \\
\hline 140 & 0.16 & 0.97 & 0.00 & 1.00 & 0.29 & 0.94 & 0.09 & 1.00 \\
\hline
\end{tabular}

Abbreviations see in Table 1.

Underlined values indicate the most valid cutoff/target values.

was significant, but the absolute change was less than the change in fat mass.

The daily average energy intake in the PreM group was $2,100 \pm 354 \mathrm{kcal}$ at 1 week before the study and it decreased significantly to $1,163 \pm 242 \mathrm{kcal}$. The PostM group significantly reduced their energy intake from $1,870 \pm 394 \mathrm{kcal}$ to $1,029 \pm 152 \mathrm{kcal}$. The daily protein intake in the PreM group was $78.1 \pm 15.1 \mathrm{~g}$, and it decreased significantly to $70.3 \pm$ $14.2 \mathrm{~g}$. In the PostM group, it decreased significantly from $86.1 \pm 33.5 \mathrm{~g}$ to $65.1 \pm 9.0 \mathrm{~g}$. The daily fat intake decreased significantly from $66.3 \pm 14.9 \mathrm{~g}$ to $33.1 \pm 9.9 \mathrm{~g}$ in the PreM group and from $56.6 \pm 20.4 \mathrm{~g}$ to $27.4 \pm 6.2 \mathrm{~g}$ in the PostM group. The daily carbohydrate intake also decreased significantly from $285.5 \pm 61.2 \mathrm{~g}$ to $147.5 \pm 31.3 \mathrm{~g}$ in the PreM group and from $272.3 \pm 94.1 \mathrm{~g}$ to $136.1 \pm 23.8 \mathrm{~g}$ in the PostM group.

The percentage of participants that exceeded each criterion of the metabolic disorders is shown in Table 3. More than $50 \%$ of the participants had a high abdominal circumference before the program (PreM, 75\%; PostM, 86\%). The most frequent disorder in both groups was hypertension, with hyper-SBP (PreM, 50\%; PostM, 67\%) and hyper DBP (PreM, 35\%; PostM, 48\%). After the program, the percentages of all disorders, except for hypo-HDLC in the PreM group, decreased.

The characteristics of the 12 provisional cutoff/target values for IFA from $30 \mathrm{~cm}^{2}$ to $140 \mathrm{~cm}^{2}$ are presented in Table 4. Sensitivities before the program ranged from 0.16 to 0.96 in the PreM group and from 0.29 to 1.00 in the PostM group. Specificities ranged from 0.04 to 0.97 for the PreM group and from 0.00 to 0.94 for the PostM group. The products obtained by multiplying the sensitivity by the specificity at each provisional value ranged from 0.04 to 0.47 in the PreM group and from 0.00 to 0.52 in the PostM group. The largest products of sensitivity and specificity were found at $80 \mathrm{~cm}^{2}(0.47)$ for the PreM group and $110 \mathrm{~cm}^{2}$ (0.52) for the PostM group. Therefore, the cutoff values with the best equilibrium between sensitivity and specificity approached $80 \mathrm{~cm}^{2}$ in the PreM group and $110 \mathrm{~cm}^{2}$ in the PostM group before weight reduction. Using the same method of analysis, the most valid target values after the weight reduction program were determined to be $60 \mathrm{~cm}^{2}$ for the PreM group and $70 \mathrm{~cm}^{2}$ for the PostM group.

\section{Discussion}

In only a few studies, attempts have been made to determine the cutoff or target value for obesity-related metabolic disorders, 19,20 In the present study the cutoff values of IFA were $80 \mathrm{~cm}^{2}$ for pre-menopausal women and $110 \mathrm{~cm}^{2}$ for post-menopausal women before weight reduction, which are similar to the $100 \mathrm{~cm}^{2}$ value considered appropriate by $\mathrm{JASSO}^{1}$ in a study that did not differentiate between preand post-menopausal women. Williams et al, in a combined study of both pre- $(n=133)$ and post-menopausal women $(\mathrm{n}=87)$, concluded that $110 \mathrm{~cm}^{2}$ was the cutoff value for IFA above which the risk of metabolic disorders increases ${ }^{20}$ Despres and Lamarche indicated that $130 \mathrm{~cm}^{2}$ of IFA was the point at which the metabolic risks increase significantly, derived from a sample of 115 males and 72 females ${ }^{19}$ Considering those findings, the cutoff values in the current study seem to be reasonable.

A difference of $30 \mathrm{~cm}^{2}$ in the cutoff values was noted between the PreM women $\left(80 \mathrm{~cm}^{2}\right)$ and the PostM women $\left(110 \mathrm{~cm}^{2}\right)$ before weight reduction. Williams et al reported that menopause has little effect on the risks of metabolic disorders, such as HDLC, TG, SBP, DBP, and TC:HDLC ratio ${ }^{20}$ although in a review by Knopp ${ }^{21}$ post-menopausal women were found to have elevated risks because of decreased estrogen contributing to increased low-density lipoprotein cholesterol (LDLC) and decreased HDLC concentrations. Hunter et $\mathrm{al}^{6}$ have also reported that postmenopausal women showed a greater IFA than pre-menopausal women and that menopausal status was significantly related to an increased risk for CHD risk factors (ie, LDLC, TC:HDLC ratio). Therefore, in the current study, the cutoff values were expected to differ according to the menopausal status of the participants. Because estrogen decreases the risk of CHD during the pre-menopausal period, perhaps counterbalancing some of the CHD risks brought on by excess $\mathrm{IF}_{21}^{21}$ further assumptions were made that the cutoff value for PreM women would be the same or even greater than that of PostM women. The ROC analyses revealed a difference of $30 \mathrm{~cm}^{2}$ between the cutoff values in each group, but the value of the PreM group was lower than that of the PostM group. The study from the Women's Health Initiative also showed that estrogen would not confer benefits for preventing CHD among women with estrogen plus progestin therapy relative to women given a placebo?2 
There seem to be other factors in addition to estrogen affecting the risk of metabolic disease; for example, aging, which correlates to an increase in IFA ${ }^{2,6,23}$ and adiponectin, ${ }^{24}$ may be a factor.

In previous studies, a cross-sectional design was used to determine an IFA cutoff value $1,19,20$ but because it is also important to determine a target IFA value for reducing the risk of metabolic disease, an intervention design was used in the current study. The IFA relates to the risk of obesityrelated metabolic disorders; therefore, we assumed that the target values after weight reduction would remain the same as before the program, but they were lower. Although the reasons for this are unclear, we speculate that once a person is suffering from a metabolic disorder, a significant reduction in IFA may not be enough in itself to ameliorate the situation.

\section{Study Limitations}

The reasons for the relatively low sensitivities and specificities derived from IFA and metabolic disorders are unclear. Some unmeasured factors, such as diet and the genetic effect of metabolic disorders, may play a part. Furthermore, homeostasis was not maintained during and just after the weight loss. Another limitation is that the number of participants was small and that the mean body mass index or IFA was not very high, although most participants were obese. Future studies should include a larger number of extremely obese participants to verify the target values for risk of IFA after weight reduction. A significant decrease in HDLC in the PreM group was found after weight reduction, which may have been caused by the diet. Hagan et $\mathrm{a}^{25}$ reported that HDLC decreased as middle-aged women lost body weight during a 12-week diet program. The significant decrease in TC could be attributed to the fact that TC includes HDLC.

In conclusion, this study presents the cutoff values for IFA in both pre- and post-menopausal obese women, as well as the target values after weight reduction, which are useful for the diagnosis of obesity-related metabolic disorders. Before weight reduction, the cutoff values with the best equilibrium were $80 \mathrm{~cm}^{2}$ for pre-menopausal women and $110 \mathrm{~cm}^{2}$ for post-menopausal women. After weight reduction, the target values shifted to $60 \mathrm{~cm}^{2}$ and $70 \mathrm{~cm}^{2}$, respectively. Using these values, persons diagnosed with visceral fat obesity can clearly see the benefits of engaging in a diet and exercise program. Furthermore, awareness of a target value makes adherence to the program more likely.

\section{Acknowledgments}

This work was supported in part by Grants-in-Aid from the Japanese Society of Physical Fitness and Sports Medicine (1998-2000), by the Tanaka Project of the Japanese Society of Physical Fitness and Sports Medicine (1998-2000), by the Tanaka Project (1999-2002) of TARA (Tsukuba Advanced Research Alliance) at University of Tsukuba, and by the 21st century COE (Center of Excellence) program, Ministry of Education, Culture, Sports, Science and Technology (2002-2003 Nishihira Project: Promotion of health and sport scientific research). The MicroDiet was provided by the Sunny Health Co Ltd.

\section{References}

1. Examination Committee of Criteria for 'Obesity Disease' in Japan, Japan Society for the Study of Obesity. New criteria for 'obesity disease' in Japan. Circ J 2002; 66: 987-992.

2. Nakamura T, Tokunaga K, Shimomura I, Nishida M, Yoshida S,
Kotani K, et al. Contribution of visceral fat accumulation to the development of coronary artery disease in non-obese men. Atherosclerosis 1994; 107: 239-246.

3. Banno K, Shiomi T, Sasanabe R, Otake K, Hasegawa R, Maekawa $\mathrm{M}$, et al. Sleep-disordered breathing in patients with idiopathic cardiomyopathy. Circ J 2004; 68: 338-342.

4. Ley CH, Lees B, Stevenson JC. Sex- and menopause-associated changes in body-fat distribution. Am J Clin Nutr 1992; 55: 950-954.

5. Björntorp PA. Sex differences in the regulation of energy balance with exercise. Am J Clin Nutr 1989; 49: 958-961.

6. Hunter GR, Kekes-Szabo T, Treuth MS, Williams MJ, Goran M, Pichon C. Intra-abdominal adipose tissue, physical activity and cardiovascular risk in pre- and post-menopausal women. Int $J$ Obes Relat Metab Disord 1996; 20: 860-865.

7. Gower BA, Nagy TR, Goran MI, Toth MJ, Poelman ET. Fat distribution and plasma lipid-lipoprotein concentrations in pre- and postmenopausal women. Int J Obes Relat Metab Disord 1998; 22: $605-$ 611.

8. Rebuffe-Scrive M, Andersson B, Olbe L, Björntorp P. Metabolism of adipose tissue in intraabdominal depots of nonobese men and women. Metabolism 1989; 38: 453-458.

9. Tokunaga K, Matsuzawa Y, Ishikawa K, Tarui S. A novel technique for the determination of body fat by computed tomography. Int $J$ Obesity 1983; 7: 437-445

10. Yoshizumi T, Nakamura T, Yamane M, Islam AH, Menju M, Yamasaki K, et al. Abdominal fat: Standardized technique for measurement at CT. Radiology 1999; 211: 283-286.

11. Igarashi K, Fujita K, Yamase T, Norita N, Okita K, Satake K, et al. Sapporo Fitness Club Trial (SFCT): Design, recruitment and implementation of a randomized controlled trial to test the efficacy of exercise at a fitness club for the reduction of cardiovascular risk factors. Circ J 2004; 68: 1199-1204.

12. The Examination Committee of Criteria for 'Metabolic Syndrome' in Japan. Criteria for 'metabolic syndrome' in Japan. J Jpn Soc Intern Med 2005; 94: 188-203 (in Japanese).

13. Tanaka K, Nakadomo F, Watanabe K, Inagaki A, Kim HK, Matsuura Y. Body composition prediction equations based on bioelectrical impedance and anthropometric variables for Japanese obese women. Am J Hum Biol 1992; 4: 739-745.

14. Brozek J, Grande F, Anderson JT, Keys A. Densitometric analysis of body composition: Revision of some quantitative assumptions. Ann NY Acad Sci 1963; 110: $113-140$.

15. Olson MS, Williford HN, Blessing DL, Greathouse R. The cardiovascular and metabolic effects of bench stepping exercise in females. Med Sci Sports Exerc 1991; 23: 1311-1317.

16. Beaver WK, Wasserman K, Whipp BJ. Improved detection of lactate threshold during exercise using a $\log -\log$ transformation. $J$ Appl Physiol 1985; 59: 1936-1940.

17. Borg G. Perceived exertion: A note on "history" and methods. Med Sci Sports 1973; 5: 90-93.

18. Hill DW, Cureton KJ, Grisham C, Collins MA. Effect of training on the rating of perceived exertion at the ventilatory threshold. Eur $J$ Appl Physiol 1987; 56: 206-211.

19. Despres JP, Lamarche B. Effects of diet and physical activity on adiposity and body fat distribution: Implications for the prevention of cardiovascular disease. Nutr Res Rev 1993; 6: 137-159.

20. Williams MJ, Hunter GR, Kekes-Szabo T, Trueth MS, Snyder S, Berland L, et al. Intra-abdominal adipose tissue cut-points related to elevated cardiovascular risk in women. Int J Obes Relat Metab Disord 1996; 20: 613-617.

21. Knopp RH. Risk factors for coronary artery disease in women. Am J Cardiol 2002; 89: 28E-35E

22. Writing Group for the Women's Health Initiative Investigators. Risks and benefits of estrogen plus progestin in healthy postmenopausal women. JAMA 2002; 288: 321-333.

23. Hernandez-Ono A, Monter-Carreola G, Zamora-Gonzalez J, Cardoso-Saldana G, Posadas-Sanchez R, Torres-Tamayo M, et al. Association of visceral fat with coronary risk factors in a populationbased sample of postmenopausal women. Int J Obes Relat Metab Disord 2002; 26: 33-39.

24. Ryo M, Nakamura T, Kihara S, Kumada M, Shibazaki S, Takahashi $\mathrm{M}$, et al. Adiponectin as a biomarker of the metabolic syndrome. Circ J 2004; 68: 975-981.

25. Hagan RD, Upton SJ, Wong L, Whittam J. The effects of aerobic conditioning and/or caloric restriction in overweight men and women. Med Sci Sports Exerc 1986; 18: 87-94. 